INPLASY

PROTOCOL

To cite: Ma et al. Effect of auricular point pressing therapy on hyperplasia of mammary glands A protocol for a systematic review and meta-analysis. Inplasy protocol 202110028. doi:

10.37766/inplasy2021.1.0028

Received: 10 January 2021

Published: 10 January 2021

Corresponding author:

Mengjie Ma

1374295753@qq.com

Author Affiliation:

Henan University of traditional Chinese Medicine

Support: Henan University of TCM.

Review Stage at time of this submission: The review has not yet started.

Conflicts of interest: None.

\section{Effect of auricular point pressing therapy on hyperplasia of mammary glands A protocol for a systematic review and meta-analysis}

\author{
Ma, MJ1; Zhang, LQ2; Wang, XL³ .
}

Review question / Objective: The aim of this meta-analysis of randomized controlled trials is to evaluate the efficacy and safety of auricular point pressing therapy of hyperplasia on mammary glands.

Condition being studied: Hyperplasia of mammary gland refers to the hyperplasia of mammary fiber and epithelial tissue, the structure degeneration of mammary duct and lobule and the growth of progressive connective tissue. The incidence rate of this disease is more common among puberty and 25-45 year old women, accounting for about $70 \%$ of the total incidence rate. Patients often appear breast pain, emotional distress and other symptoms.Mammary gland hyperplasia belongs to the category of "breast fetish" in traditional Chinese medicine, which is mainly caused by stagnation of liver qi, spleen injury caused by thought or imbalance of Chong and Ren, and stagnation of qi and blood stasis.

INPLASY registration number: This protocol was registered with the International Platform of Registered Systematic Review and Meta-Analysis Protocols (INPLASY) on 10 January 2021 and was last updated on 10 January 2021 (registration number INPLASY202110028).

\title{
INTRODUCTION
}

Review question / Objective: The aim of this meta-analysis of randomized controlled trials is to evaluate the efficacy and safety of auricular point pressing therapy of hyperplasia on mammary glands.
Condition being studied: Hyperplasia of mammary gland refers to the hyperplasia of mammary fiber and epithelial tissue, the structure degeneration of mammary duct and lobule and the growth of progressive connective tissue. The incidence rate of this disease is more common among 
puberty and 25-45 year old women, accounting for about $70 \%$ of the total incidence rate. Patients often appear breast pain, emotional distress and other symptoms.Mammary gland hyperplasia belongs to the category of "breast fetish" in traditional Chinese medicine, which is mainly caused by stagnation of liver qi, spleen injury caused by thought or imbalance of Chong and Ren, and stagnation of qi and blood stasis.

\section{METHODS}

Participant or population: Regardless of nationality, age, gender, occupation, participants met the clinical diagnostic criteria for breast hyperplasia were included. Cases associated with serious illness, pregnancy, lactation period were excluded.

Intervention: The experimental group was treated with auricular point pressing therapy. The control group was treated with placebo, drugs, or other alternative therapy.

Comparator: There is no exclusion based on comparator method for this review, and the patients could be treated with any forms of control group.

Study designs to be included: We will only include randomized controlled trial (RCTs), non-RCTs, quasi-RCTs, reviews, and other types of studies will be excluded.

Eligibility criteria: We will only include randomized controlled trial (RCTs), nonRCTs, quasi-RCTs, reviews, and other types of studies will be excluded.Regardless of nationality, age, gender, occupation, participants met the clinical diagnostic criteria for breast hyperplasia were included. Cases associated with serious illness, pregnancy, lactation period were excluded. The experimental group was treated with auricular point pressing therapy. The control group was treated with placebo, drugs, or other alternative therapy.There is no exclusion based on comparator method for this review, and the patients could be treated with any forms of control group.The main outcome(s) are: complete disappearance of pain symptoms; diameter and area of breast mass;hormone levels of luteinizing hormone, 17aestradiol, prolactin, and progesterone。the additional outcome(s) are: abnormal menstruation; mood changes; adverse reaction; Patient satisfaction.

Information sources: PubMed, Web of Science, Cochrane Library, EMBASE, Wan fang Database, Chinese Scientific Journal Database, CNKI, VIP and Chinese Biomedical Literature Database were systematically searched by computer. The retrieval date was up to January 10, 2021. The details of PubMed's search strategy are illustrated in Table 1, while similar search strategies are applied for other electronic databases.

Main outcome(s): 1. complete disappearance of pain symptoms; 2 . diameter and area of breast mass; 3. hormone levels of luteinizing hormone, 17aestradiol, prolactin, and progesterone.

Quality assessment / Risk of bias analysis: The quality evaluation of literatures is based on Cochrane handbook system evaluation manual. The items of quality including random sequence generation method, allocation concealment, blinding of subjects, and intervention providers, blinding of outcome evaluators, completeness of result data, selective result reporting and other sources of bias.According to the criteria, the included studies were divided into 3 levels: low, medium, and high.

Strategy of data synthesis: The Revman $\mathbf{5 . 3}$ software will be used to perform all statistical analyses. and the heterogeneity was detected by $\mathbf{Q}$ test and 12 test. If $P>$ $0.1,12<50 \%$, there is no significant heterogeneity between the included studies, fixed effect model will be used ; If $P<0.1$ and $I 2 \geq 50 \%$, it indicates that there is obvious heterogeneity among the included studies. We will use random effect model and subgroup analysis, sensitivity 
analysis to analyze the sources of heterogeneity to eliminate its impact.

Subgroup analysis: When there is obvious heterogeneity ( such as age, gender, different types of intervention, publication year, etc. ), subgroup analysis and metaregression analysis will be conducted to identify the sources of heterogeneity.

Sensibility analysis: For the quality analysis, we will conduct a sensitivity analysis of main outcomes to test the stability of the results of meta-analysis.

Country(ies) involved: China.

Keywords: auricular point pressing, hyperplasia of mammary glands, metaanalysis, systematic review.

Contributions of each author:

Author 1 - Mengjie Ma.

Author 2 - Liuqiao Zhang.

Author 3 - Xiangli Wang.

Conflicts of interest: We declare that we have no financial and personal relationships with other people or organizations that can inappropriately influence our work, there is no professional or other personal interest of any nature or kind in any product, service and/or company that could be construed as influencing the position presented in, or the review. 